\title{
Three-dimensional echocardiography for left ventricular quantification: fundamental validation and clinical applications
}

\author{
J. A. van der Heide • S. A. Kleijn • M. F. A. Aly • \\ J. Slikkerveer • O. Kamp
}

Published online: 17 May 2011

(C) The Author(s) 2011. This article is published with open access at Springerlink.com

\begin{abstract}
One of the earliest applications of clinical echocardiography is evaluation of left ventricular (LV) function and size. Accurate, reproducible and quantitative evaluation of LV function and size is vital for diagnosis, treatment and prediction of prognosis of heart disease. Early three-dimensional (3D) echocardiographic techniques showed better reproducibility than two-dimensional (2D) echocardiography and narrower limits of agreement for assessment of LV function and size in comparison to reference methods, mostly cardiac magnetic resonance (CMR) imaging, but acquisition methods were cumbersome and a lack of user-friendly analysis software initially precluded widespread use. Through the advent of matrix transducers enabling real-time three-dimensional echocardiography (3DE) and improvements in analysis software featuring semi-automated volumetric analysis, 3D echocardiography evolved into a simple and fast imaging modality for everyday clinical use. 3DE provides the possibility to evaluate the entire LV in three spatial dimensions during the complete cardiac cycle, offering a more accurate and complete quantitative evaluation the LV. Improved efficiency in acquisition and analysis may provide clinicians with important diagnostic information within minutes. The current
\end{abstract}

Drs Kleijn and Van der Heide contributed equally to this paper.

J. A. van der Heide · S. A. Kleijn · M. F. A. Aly · J. Slikkerveer •

O. Kamp $(\triangle)$

Department of Cardiology 5F 003,

VU University Medical Center,

De Boelelaan 1117,

1081 HV Amsterdam, the Netherlands

e-mail: o.kamp@vumc.nl

S. A. Kleijn · J. Slikkerveer · O. Kamp Interuniversity Cardiology Institute of the Netherlands (ICIN), Utrecht, the Netherlands article reviews the methodology and application of 3DE for quantitative evaluation of the $\mathrm{LV}$, provides the scientific evidence for its current clinical use, and discusses its current limitations and potential future directions.

Keywords Three-dimensional echocardiography. Left ventricle · Dyssynchrony · Endocardial contour . Speckle tracking

\section{Introduction}

One of the earliest and most common applications of clinical echocardiography is evaluation of left ventricular (LV) function and size. Accurate, reproducible and quantitative evaluation of LV function and size is vital for diagnosis, treatment and prediction of prognosis of heart disease. Early three-dimensional (3D) echocardiographic techniques showed better reproducibility than two-dimensional (2D) echocardiography and narrower limits of agreement for assessment of LV function and size in comparison with reference methods, mostly cardiac magnetic resonance (CMR) imaging, but acquisition methods were cumbersome and a lack of userfriendly analysis software initially precluded widespread use [1-3]. Through the advent of matrix transducers enabling real-time $3 \mathrm{D}$ echocardiography $(3 \mathrm{DE})$ and impressive improvements in analysis software featuring semi-automated volumetric analysis, $3 \mathrm{DE}$ evolved into a simple and fast imaging modality for everyday clinical use. 3DE provides the possibility to evaluate the entire LV in three spatial dimensions during the complete cardiac cycle, offering a more accurate and complete quantitative evaluation of the LV [4-7]. Improved efficiency in acquisition and analysis may provide clinicians with important diagnostic information within minutes. 
The current article reviews the methodology and application of $3 \mathrm{DE}$ for quantitative evaluation of the $\mathrm{LV}$, provides the scientific evidence for its current clinical use, and discusses its current limitations and potential future directions.

\section{Methodology}

\section{Technology}

3DE has been made possible in particular by the development of matrix transducers. A matrix transducer contains thousands of piezoelectric elements in a 2D array, which can be electronically steered in multiple directions very rapidly, enabling the acquisition of a real-time 3D volume dataset. Such a volume dataset has a pyramidal shape with a curved base and is approximately $30^{\circ} \times 60^{\circ}$, depending on which manufacturer's hardware is used. This is more than adequate for visualisation of different structures within the heart such as valves and masses. Until recently, inclusion of the entire LV within a dataset necessitated an automated ECG-triggered capture of multiple consecutive real-time datasets (usually four to seven) during briefly held respiration and electronically stitching these datasets together. The latest generation of 3D scanners provide acquisition of a 'full volume' at a frame rate of 40-50 Hz. Either method results in a 'full-volume' dataset of up to $90^{\circ} \times 110^{\circ}$, which in general is sufficient to allow for accurate analysis of the LV. The use of more sub-volumes results in higher line density and higher resolution. However, it also increases the chance of stitching artifacts due to potentially erroneous ECG triggering causing unsynchronised subvolumes. Until recently, this precluded $3 \mathrm{D}$ analysis in patients with irregular heart rhythms such as atrial fibrillation. Fortunately, one of the more recent developments in $3 \mathrm{DE}$ is the possibility of acquiring a full-volume dataset within a single heartbeat, obviating this limitation by providing instantaneous real-time volumetric imaging of the entire LV and decreasing acquisition time. A recent comparison between multiple consecutive real-time acquisitions, twobeat and single-beat acquisitions demonstrated a significantly lower frame rate in single-beat acquisitions with, as a consequence, underestimation of ejection fraction (EF) [8]. The two-beat modality provided similar accuracy in LV volume and EF measurements and may be preferred due to fewer stitching artifacts. In atrial fibrillation, however, single-beat acquisition may be superior because absence of stitching artifacts may be more important than image quality deterioration [9].

\section{Acquisition}

After positioning the transducer accurately to include the whole $\mathrm{LV}$ into the $3 \mathrm{D}$ volume, a single $3 \mathrm{D}$ acquisition generally takes less than $10 \mathrm{~s}$. Although all standard acquisition windows for echocardiography are available and useful for 3D acquisitions, the ideal and generally preferred approach for acquisition of the LV is from the apical window. Depending on the shape of the heart and its position within the chest, a more off-axis position may be appropriate to ensure the acquisition of the entire LV. To guarantee optimal image quality, transducer frequency and overall gain should be adjusted accordingly. Typically, the gain setting should be somewhat higher than used for $2 \mathrm{D}$ echocardiography to get the most favourable result. In addition, the smallest possible dataset encompassing the whole LV should be acquired to ensure maximal temporal and spatial resolution. Finally, it is important to ascertain that the acquisition is made during breath hold to minimise the risk of breathing artifacts. In multi-beat acquisitions, stitching artifacts can easily be detected in the transversal plane.

\section{Visualisation}

Concurrently with the acquisition of a 3D dataset, it is volume rendered within the echocardiography system, resulting in a $3 \mathrm{D}$ volume dataset which can be rotated and viewed from any angle. In combination with the ability to crop part of the dataset away, it is possible to visualise the anatomy and function of the LV in great detail. Cropping a $3 \mathrm{D}$ volume can be done by utilising a 'crop-box' and by use of an 'anyplane' mode. With the first method the 3D dataset is displayed within a box, the sides of which can be selected and cropped away. The more conventional 2D long-axis and short-axis views can be inspected easily and rapidly this way but ultimately any long-axis or short-axis view can be chosen. The 'anyplane' mode enables cropping of the $3 \mathrm{D}$ volume from every conceivable orientation, providing unlimited sections of the LV. Although the interpretation of such unconventional cut-planes can initially be difficult, the learning curve is relatively steep due to the $3 \mathrm{D}$ nature of the acquired images.

\section{Analysis}

Most vendors offer software packages for both online and offline quantitative analysis of the LV. Most of these commercially available software packages provide the possibility to check for stitching artifacts in a transversal plane using the slicing (particularly multislice) function that can also be used to analyse wall motion and distribution of hypertrophy. Furthermore, certain anatomical landmarks such as the mitral annulus and apex are identified in multiple conventional 2D planes. Thereafter, the software semi-automatically detects and traces the endocardial border of the LV during the cardiac cycle, with a possibility 
to perform manual corrections as needed [10]. Subsequently the software creates a cast of the LV, allowing comprehensive volumetric analysis of LV function and morphology. Not only LV volumes and ejection fraction (EF) can automatically be calculated, but time-volume curves can be generated to evaluate segmental LV function and the presence of dyssynchrony. Figure 1 is a screen shot of the work flow of one of the commercially available software packages. Finally, the segmental contraction pattern can be displayed graphically through parametric imaging in a dynamic 'bulls eye' display (an example is shown in Fig. 2), providing information on segmental volume changes and timing of segmental contraction, which may be of particular interest in guiding optimal LV lead placement for cardiac resynchronisation therapy (CRT).
Feasibility

An adequate echocardiographic image quality is a prerequisite for meaningful 3DE and most authors use image quality as an inclusion criterion. Four articles provide information about image quality in an unselected population [9, 11-13]. In a total of 270 patients, 162 had good or optimal image quality, 51 had average image quality, and 41 had poor image quality.

Furthermore we would like to comment that in our experience, semi-automated endocardial contour detection is influenced negatively by the presence of chordae tendiniae, papillary muscles and sub-valvular mitral calcification, even in patients with a good image quality necessitating doing some manual corrections of the automated endocardial contour in the majority of patients.

Fig. 1 Example of the work flow of one of the commercially available software packages. Top, left and right: two perpendicular long-axis images of the LV. The endocardial contour is detected semi-automatically and is projected on the image. Centre left: a third perpendicular short-axis image, again with the endocardial contour. Note that this short-axis image was made with the ultrasound transducer in the apical position. Centre right: The LV 'cast' is shown with colourcoding of individual LV segments. Bottom: For each LV segment, a time-volume curve is generated. Regional wall motion and dyssynchrony can be appreciated easily

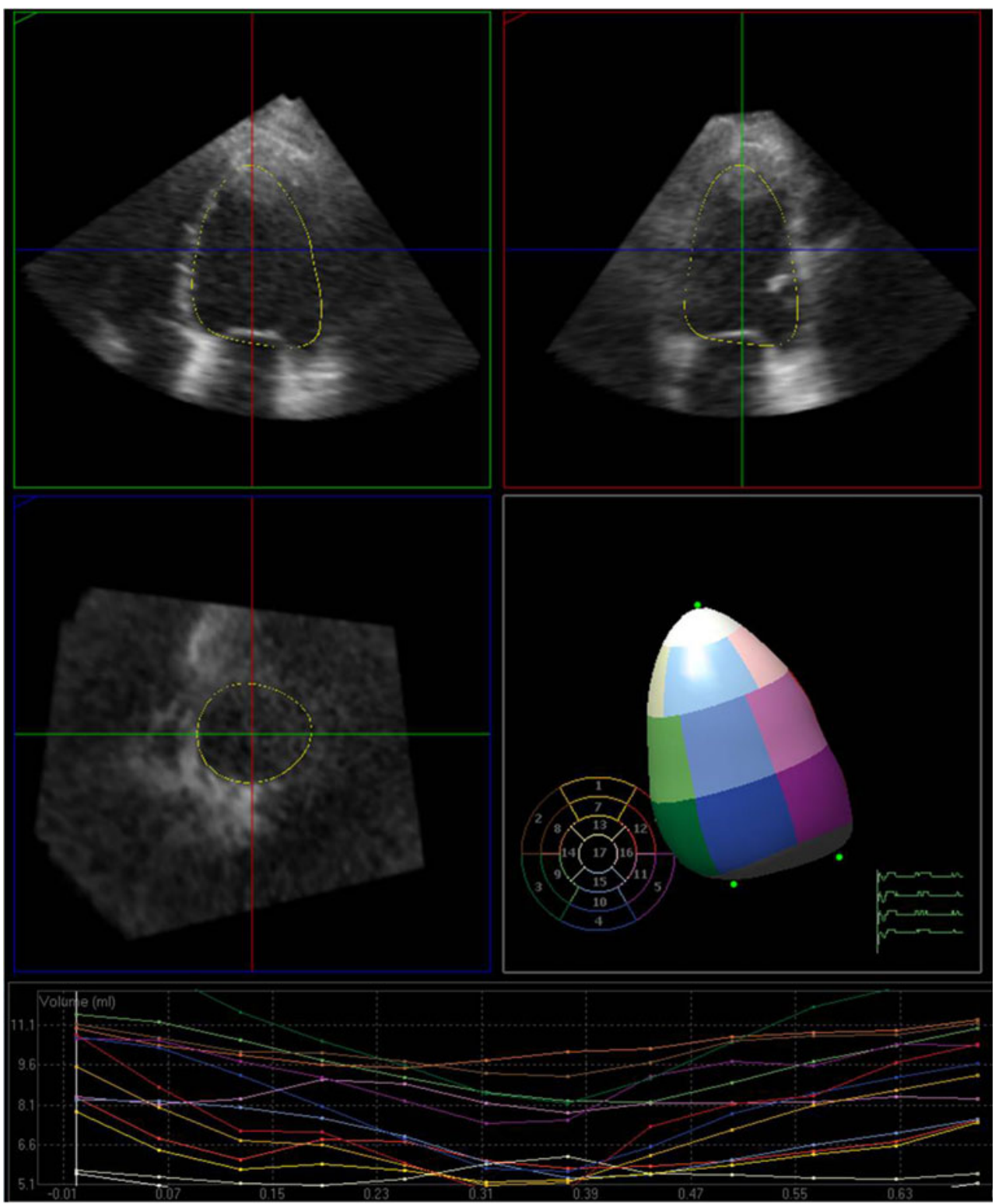




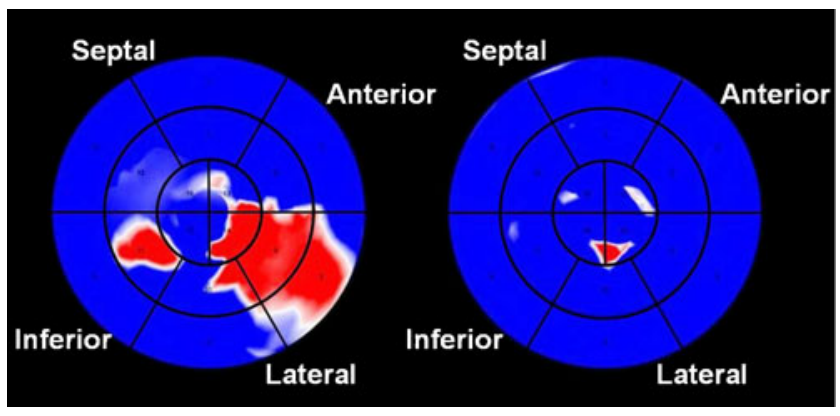

Fig. 2 Parametric images derived from three-dimensional datasets. Images of a patient are shown with CRT turned off (left) and on (right), respectively. The LV is divided into 16 segments as described by the American Society of Echocardiography. Colour coding is used to represent regional time-to-minimum systolic volume, with red indicating late activation. In the left hand map, the lateral wall is identified as the latest activated LV region without CRT (left). As can be appreciated in the right hand map by the overall homogenous blue colour indicating the absence of large regions with delayed contraction, synchronicity is re-established when CRT is applied (right)

Recently, a new matrix transducer (X Matrix X5-1, Philips, Andover, MA) was introduced that has a smaller footprint and allows acquisition of both 2D images and 3D datasets without the need to change the probe providing a higher temporal and spatial resolution and improving the feasibility and quality of acquisitions. In addition to the need to improve image quality, wider angle acquisition is also indispensable for adequate visualisation of the entire heart in one volume in severely enlarged ventricles. Further improvement in border detection and speckle tracking algorithms is necessary to obviate the need for offline analysis. Moreover, further development of DICOM algorithms, which allow to display and analyse 3D datasets independently on vendor specific software, is also needed.

\section{Clinical applications}

\section{LV volumes and function}

In evaluation of LV function, 2D echocardiography has its own limitations, mainly that quantitative measurements such as Simpson's biplane summation-of-disks method for LV volume measurement are based on assumptions of symmetrical LV geometry that are inherently unreliable. These assumptions as well as the unintentional use of foreshortened views account for the relative inaccuracy and poor reproducibility of these techniques. 3DE obviates both these limitations, and therefore measurements of LV volumes and $\mathrm{EF}$ are significantly more accurate and reproducible than conventional 2D echocardiography when compared with CMR as the reference technique (Figs. 3 and 4) [6-30]. Unsurprisingly, studies that only included patients with good echocardio- graphic image quality $[6,7,21,22,30]$ showed better agreement than those who did not [13, 19, 23]. Furthermore, studies using semi-automated endocardial contour analysis $[6$, $13,26,28,29]$ in $3 \mathrm{DE}$ generally showed better agreement than those using manual contour tracing [19, 23, 27]. An exception to the rule is an early study by Kühl et al. [4] published in 2004, but it is likely that semi-automatic contour detection software has substantially improved since then. Finally, two studies focusing on potential differences between semi-automated contour tracing algorithms found some of the differences to be statistically significant, but clinically irrelevant $[22,30]$.

Despite the high correlation with CMR as a reference technique, most studies showed significant underestimation of 3DE-derived LV volumes, irrespective of the method of analysis $[19,23,26]$. The potential reasons for this systematic underestimation of $\mathrm{LV}$ volumes by $3 \mathrm{DE}$ as compared with CMR were identified in a recent multicentre study by Mor-Avi et al [25]. They concluded that 3DEderived LV volumes are underestimated in most patients because, unlike CMR, 3DE imaging cannot differentiate between the myocardium and trabeculae. Therefore, in most patients the endocardial contour is traced along the innermost endocardial border by 3DE, i.e. excluding the trabeculae from the LV cavity. By convention, however, the endocardial contour is traced along the outermost endocardial border by CMR, i.e. including the trabeculae in the LV cavity. To minimise the difference between the two modalities, tracing the endocardium to include trabeculae in the LV cavity is recommended for 3DE. In addition to tracing the endocardium beyond the visible interface, sufficient experience [25] and good image quality [31] are the most important factors to achieve accurate and reproducible 3DE quantification of LV volumes. Finally, if image quality is poor, multiple studies have shown that echo contrast agents can be of incremental value to improve the accuracy and reproducibility of LV volume and function measurements $[13,23,32]$.

The superiority of $3 \mathrm{DE}$ over $2 \mathrm{DE}$ in clinical decisionmaking was proven in a study comparing the clinical impact of $3 \mathrm{DE}$ versus $2 \mathrm{DE}$ in different clinical scenarios. This study concluded that measurement of LV volumes and EF by $3 \mathrm{DE}$ is clinically feasible and has the potential to significantly alter clinical decision-making [33]. Further studies and more robust outcome data on defining 3DE cut-offs for clinical decision are still needed to confirm such clinical superiority on definite and clinically meaningful endpoints to be part of the routine clinical use.

\section{LV dyssynchrony}

Cardiac resynchronisation therapy has been shown to improve LV function, survival and quality of life. However, 

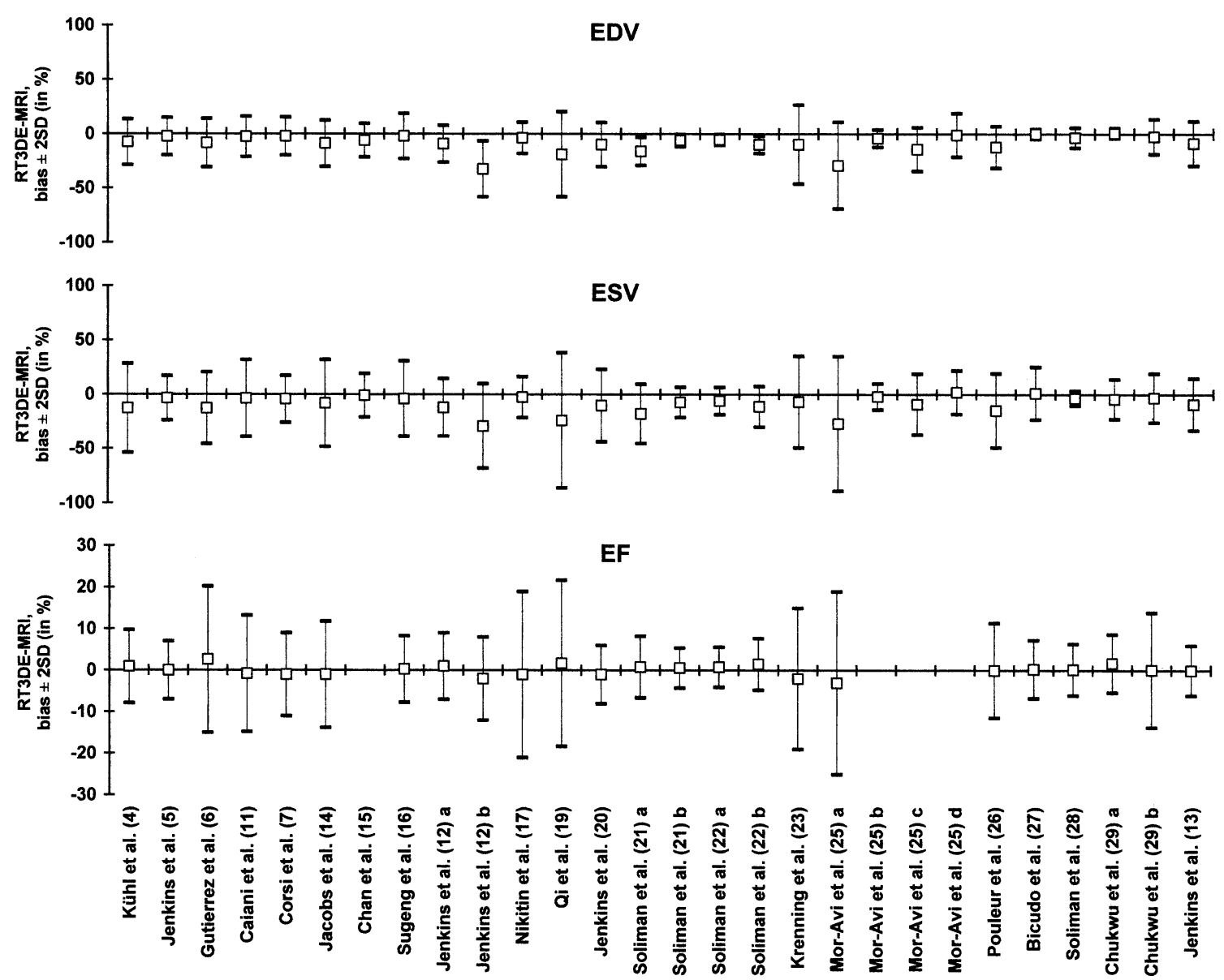

Fig. 3 LV volumes and EF measurements by 3DE: comparison with CMR. In total, we identified 21 studies including 1040 patients (747 males, $60 \pm 9$ years) in which LV volumes and ejection fraction as measured by 3DE were compared with CMR as the reference method [47, 11-17, 19-23, 25-29]. Bland-Altman statistics are shown for EDV, $\mathrm{ESV}$, and EF by $3 \mathrm{DE}$ in comparison with CMR as the reference method. Mean difference \pm 2 standard deviations are shown as a percentage of the mean value, as measured by $3 \mathrm{DE}$. Studies are shown in chronological order, with the most recent publications to the right. Jenkins et al. [12]: $a$ TomTec offline, $b$ QLAB online; Soliman et al. [21]: $a$ multiplane interpolation, $b$ full-volume reconstruction; Soliman et al. [22]: $a$ TomTec 4D LV-Analysis ver. 2.0, $b$ QLAB V4.2; Mor-Avi et al. [25]: $a$ CMR short-axis vs. 3DE long-axis image analysis, $b \mathrm{CMR}$ and $3 \mathrm{DE}$ long-axis image analysis, $c$ trabeculae included in LV volume in CMR analysis, $d$ trabeculae excluded from LV volume in CMR analysis; Chukwu et al. [29]: $a$ normal volunteers, $b$ myocardial infarction patients accurate prediction of response to pacing remains difficult as approximately $30 \%$ of patients selected with current selection criteria do not respond to CRT [34]. A low correlation exists between LV mechanical dyssynchrony and QRS duration, and mechanical dyssynchrony might be a better predictor of response to CRT than QRS duration. At present, tissue Doppler imaging (TDI) is the mostly used echocardiographic technique for quantification of mechanical dyssynchrony $[35,36]$. However, TDI can only provide information on the systolic myocardial motion of the basal and mid-segments, in the longitudinal direction. Because its reproducibility is relatively low and no TDI-based dyssynchrony parameter has proven to be useful for the prediction of response to CRT in a large clinical trial [37], the need for reliable alternative echocardiographic parameters of dyssynchrony has been recognised. $3 \mathrm{DE}$ offers information on systolic contraction based on inward endocardial motion, providing a complete evaluation of intraventricular mechanical dyssynchrony of all LV segments simultaneously. The site of maximal mechanical delay can be visualised in a polar map. In addition, quantitative dyssynchrony analysis is possible by measuring time needed for each individual segment to reach minimum systolic volume. The standard deviation of the dispersion in time from the onset of QRS complex to minimal regional volume of all segments can be used as a parameter of LV dyssynchrony.

There are conflicting reports on the correlation between LV dyssynchrony measured by TDI and 3DE methods, ranging from fair to poor agreement [38-43]. In large part this has been attributed to the fact that $3 \mathrm{DE}$ and TDI provide distinctly different measures of ventricular motion. When both techniques were directly compared for 

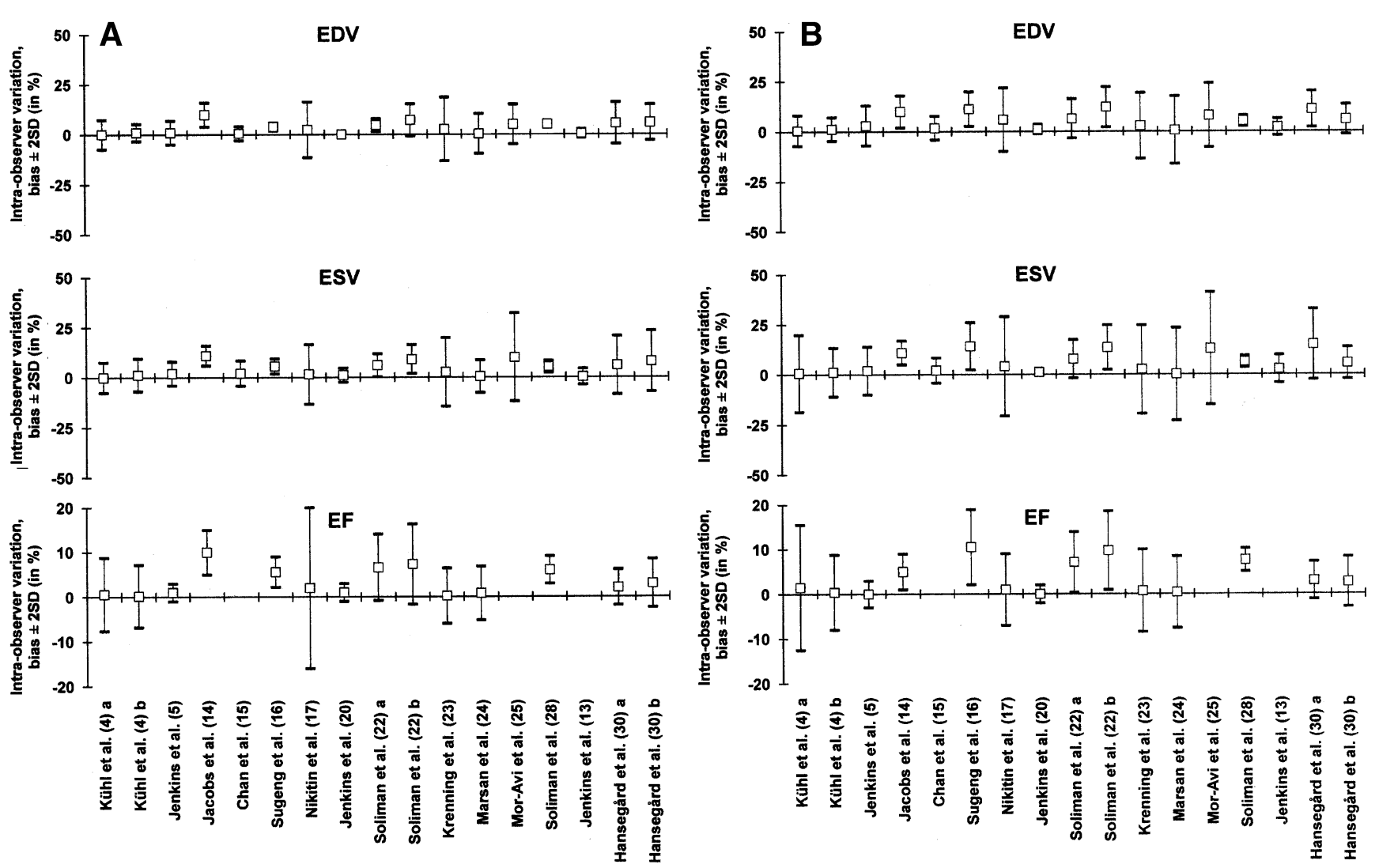

Fig. $4 \mathrm{LV}$ volumes and function measurements by $3 \mathrm{DE}$ : a intraobserver variability; b inter- observer variability. Legend: To study the reproducibility of LV volumes and EF measurements, we identified 16 studies including 407 patients in which reproducibility of these measurements by 3DE was assessed [4, 5, 13-17, 20, 22-25, 28, 30]. Studies are shown in chronological order, with the most recent

publications to the right. Kühl et al. [4]: $a$ TomTec CardioView RT semi-automated analysis, $b$ TomTec CardioView RT manual analysis; Soliman et al. [22]: $a$ TomTec 4D LV-Analysis ver. 2.0, $b$ Philips QLAB V4.2; Hansegård et al. [30]: $a$ TomTec 4D LV-Analysis ver. 2.2, $b$ GE 4DLVQ software, EchoPAC ver. 108.1.0

prediction of volumetric and clinical response to CRT, results clearly showed that 3DE has better sensitivity and specificity to predict response to CRT than TDI [43]. Three other studies demonstrated that 3DE-derived SDI was highly predictive of both the acute and chronic response $[24,42,44]$. However, currently there is no agreement on

cut-off values for the prediction of response to CRT and a larger multicentre study assessing reproducibility and predictive value for clinical endpoints is warranted. Reference values and reproducibility of LV dyssynchrony in normal volunteers and in various groups of patients are given in Tables 1 and 2. These values are highly variable and are

Table 1 Reference values for 3DE-derived systolic dyssynchrony index (SDI)

\footnotetext{
${ }^{a}$ Patients with dilated cardiomyopathy without left bundle branch block, ${ }^{\mathrm{b}}$ patients with dilated cardiomyopathy and left bundle branch block
}

\begin{tabular}{lccccc}
\hline Study (reference) & Segments & $\begin{array}{l}\text { SDI (\%) in healthy } \\
\text { volunteers }\end{array}$ & $\mathrm{n}$ & $\begin{array}{l}\text { SDI (\%) in chronic } \\
\text { heart failure patients }\end{array}$ & $\mathrm{n}$ \\
\hline Kapetanakis et al. [38] & 16 & $3.5 \pm 1.6$ & 78 & $15.7 \pm 6.7$ & 44 \\
Soliman et al. [42] & 17 & $4.1 \pm 2.2$ & 60 & $13.4 \pm 8.1$ & 84 \\
Kleijn et al. [43] & 16 & $3.7 \pm 2.6$ & 30 & $9.7 \pm 4.0$ & 60 \\
Van Dijk et al. [45] & 16 & $5.6 \pm 3.6$ & 16 & $12.8 \pm 4.8$ & 23 \\
Delgado et al. [47] & 17 & $1.5 \pm 0.7$ & 10 & $14.3 \pm 7.5$ & 22 \\
Gimenes et al. [48] & 16 & $1.6 \pm 1.0$ & 120 & & 35 \\
Liodakis et al. [49] & 16 & $9.8 \pm 2.1$ & 35 & $17.3 \pm 4.0$ & 16 \\
Sonne et al. [51] & 16 & $2.2 \pm 0.9$ & 16 & $9.1 \pm 1.1^{\mathrm{a}}$ & $8.7 \pm 2.9^{\mathrm{b}}$ \\
& & & & & 16 \\
\hline
\end{tabular}


Table 2 Intra- and inter-observer variability of SDI measurement by $3 \mathrm{DE}$

\begin{tabular}{lcccc}
\hline Study (reference) & Average value & $\begin{array}{l}\text { Intra-observer } \\
\text { variability (bias } \pm \text { 2SD) }\end{array}$ & $\begin{array}{l}\text { Inter-observer variability } \\
\text { (bias } \pm \text { 2SD) }\end{array}$ & $\mathrm{n}$ \\
\hline Marsan et al. [24] & 7.3 & $0.0 \pm 0.5$ & $0.1 \pm 1.0$ & 20 \\
Kapetanakis et al. [38] & 6.6 & $0.6 \pm 4.9$ & $0.5 \pm 2.9$ & 20 \\
Marsan et al. [41] & 8.4 & & $0.1 \pm 1.0$ & 20 \\
Van Dijk et al. [45] & 8.9 & $0.0 \pm 7.6$ & $1.9 \pm 5.0$ & 20 \\
Marsan et al. [46] & 7.8 & $0.0 \pm 0.4$ & $0.1 \pm 2.0$ & 20 \\
Delgado et al. [47] & 7.9 & $0.0 \pm 5.0$ & $0.4 \pm 6.4$ & 18 \\
Soliman et al. [50] & 11.5 & $0.3 \pm 3.4$ & $0.3 \pm 5.1$ & 50 \\
\hline
\end{tabular}

based on relatively small studies in which varying software was used [24, 38, 41-51].

Finally, the importance of optimal LV pacing lead position was emphasised in a 3DE study [52] comparing response to CRT, defined as increase in oxygen uptake and decrease in LV volume, in patients with the LV pacing lead at the segment with the maximum mechanical delay to patients with the LV pacing lead at other segments. Response was optimal in patients with optimal LV pacing lead position as assessed by 3DE, and decreased significantly with increasing distance between optimal pacing site and achieved pacing site. In this regard, 3DE may prove to be useful for the optimisation of LV lead placement [39, 52]. In contrast, a smaller study [53] with a similar design found that implantation of the LV lead in the most delayed LV segment determined by 3DE did not result in additional improvement in symptoms or LV function.

\section{Future perspectives}

Semi-automated endocardial contour detection provides information of systolic contraction based on 3D endocardial excursion only. In contrast, speckle tracking is an angleindependent technique that can quantitatively evaluate myocardial deformation or strain in multiple directions by tracking the movement of natural acoustic markers of the myocardium known as speckles which appear on grey-scale images. When applied to 2D echocardiography images, however, speckle tracking is significantly hampered by the intrinsic nature of its 2D methodology. Not only foreshortened views and geometric assumptions limit the applicability of $2 \mathrm{D}$ speckle tracking, but out-of-plane motion of speckles during the cardiac cycle also result in suboptimal tracking and considerable noise, reducing the accuracy and reproducibility of its quantitative evaluation of strain.

Recently, 3D speckle tracking has been introduced to overcome these limitations. With 3D speckle tracking, the complex LV wall motion can be assessed in all three spatial dimensions and there is no loss of information because the speckles do not move in and out of the volume. Measurements are based on regions of interest rather than (semiautomated) contour detection. 3D speckle tracking measurements of LV volumes shows higher correlation with CMR, smaller biases, and narrower limits of agreement compared with 2D speckle tracking [54]. LV twist has been reliably measured, but as yet in healthy volunteers only [55]. In LV mechanical dyssynchrony, semiautomatic assessment is reasonably reproducible and a clear difference was demonstrated between RV apical and biventricular pacing [56]. The ability to identify the site of latest mechanical activation may aid in optimisation of biventricular lead placement [57]. Additional studies will have to validate the use of this novel technology for different clinical purposes and establish its incremental value compared with volumetric endocardial contour tracing.

\section{Conclusions}

In the last two decades the technology behind 3DE has greatly evolved, continually expanding its clinical applications. Ample evidence suggests that LV volumes and function measurements by $3 \mathrm{DE}$ have closer limits of agreement with CMR measurements as a reference method and better reproducibility than $2 \mathrm{D}$ echocardiography. 3DE assessment of LV dyssynchrony shows great potential. Future advancements in hardware will facilitate the acquisition of wide-angle, single-cycle pyramidal datasets with higher spatial and temporal resolution. Automatic analysis of endocardial contours and speckle tracking will enable fast online measurements that are accurate and reproducible and will ensure its integration into the routine echocardiographic examination.

Open Access This article is distributed under the terms of the Creative Commons Attribution Noncommercial License which permits any noncommercial use, distribution, and reproduction in any medium, provided the original author(s) and source are credited. 


\section{References}

1. Buck T, Hunold P, Wentz KU, et al. Tomographic three-dimensional echocardiographic determination of chamber size and systolic function in patients with left ventricular aneurysm: comparison to magnetic resonance imaging, cineventriculography, and twodimensional echocardiography. Circulation. 1997;96:4286-97.

2. Chuang ML, Hibberd MG, Salton CJ, et al. Importance of imaging method over imaging modality in noninvasive determination of left ventricular volumes and ejection fraction: assessment by two- and three-dimensional echocardiography and magnetic resonance imaging. J Am Coll Cardiol. 2000;35:477-84.

3. Mannaerts HF, Van Der Heide JA, Kamp O, et al. Quantification of left ventricular volumes and ejection fraction using freehand transthoracic three-dimensional echocardiography: comparison with magnetic resonance imaging. J Am Soc Echocardiogr. 2003;16:101-9.

4. Kuhl HP, Schreckenberg M, Rulands D, et al. High-resolution transthoracic real-time three-dimensional echocardiography: quantitation of cardiac volumes and function using semi-automatic border detection and comparison with cardiac magnetic resonance imaging. J Am Coll Cardiol. 2004;43:2083-90.

5. Jenkins C, Bricknell K, Hanekom L, et al. Reproducibility and accuracy of echocardiographic measurements of left ventricular parameters using real-time three-dimensional echocardiography. J Am Coll Cardiol. 2004;44:878-86.

6. Gutierrez-Chico JL, Zamorano JL, Perez de Isla L, et al. Comparison of left ventricular volumes and ejection fractions measured by threedimensional echocardiography versus by two-dimensional echocardiography and cardiac magnetic resonance in patients with various cardiomyopathies. Am J Cardiol. 2005;95:809-13.

7. Corsi C, Lang RM, Veronesi F, et al. Volumetric quantification of global and regional left ventricular function from real-time threedimensional echocardiographic images. Circulation. 2005;112:116170 .

8. Macron L, Lim P, Bensaid A, et al. Single-beat versus multibeat realtime 3D echocardiography for assessing left ventricular volumes and ejection fraction: a comparison study with cardiac magnetic resonance. Circ Cardiovasc Imaging. 2010 Jul 1;3(4):450-5. Epub 2010 Apr 30.

9. Shahgaldi K, Manouras A, Abrahamsson A, et al. Threedimensional echocardiography using single-heartbeat modality decreases variability in measuring left ventricular volumes and function in comparison to four-beat technique in atrial fibrillation. Cardiovasc Ultrasound. 2010;8(1):45.

10. Muraru D, Badano LP, Piccoli G, et al. Validation of a novel automated border-detection algorithm for rapid and accurate quantitation of left ventricular volumes based on three-dimensional echocardiography. Eur J Echocardiogr. 2010;11(4):359-68.

11. Caiani EG, Corsi C, Zamorano J, et al. Improved semiautomated quantification of left ventricular volumes and ejection fraction using 3-dimensional echocardiography with a full matrix-array transducer: comparison with magnetic resonance imaging. J Am Soc Echocardiogr. 2005;18:779-88.

12. Jenkins C, Chan J, Hanekom L, et al. Accuracy and feasibility of online 3-dimensional echocardiography for measurement of left ventricular parameters. J Am Soc Echocardiogr. 2006;19:1119-28.

13. Jenkins C, Moir S, Chan J, et al. Left ventricular volume measurement with echocardiography: a comparison of left ventricular opacification, three-dimensional echocardiography, or both with magnetic resonance imaging. Eur Heart J. 2009;30:98-106.

14. Jacobs LD, Salgo IS, Goonewardena S, et al. Rapid online quantification of left ventricular volume from real-time threedimensional echocardiographic data. Eur Heart J. 2006;27:460-8.

15. Chan J, Jenkins C, Khafagi F, et al. What is the optimal clinical technique for measurement of left ventricular volume after myocardial infarction? A comparative study of 3-dimensional echocardiography, single photon emission computed tomography, and cardiac magnetic resonance imaging. J Am Soc Echocardiogr. 2006;19:192-201.

16. Sugeng L, Mor-Avi V, Weinert L, et al. Quantitative assessment of left ventricular size and function: side-by-side comparison of realtime three-dimensional echocardiography and computed tomography with magnetic resonance reference. Circulation. 2006;114:654-61.

17. Nikitin NP, Constantin C, Loh PH, et al. New generation 3dimensional echocardiography for left ventricular volumetric and functional measurements: comparison with cardiac magnetic resonance. Eur J Echocardiogr. 2006;7:365-72.

18. Malm S, Frigstad S, Sagberg E, et al. Real-time simultaneous triplane contrast echocardiography gives rapid, accurate, and reproducible assessment of left ventricular volumes and ejection fraction: a comparison with magnetic resonance imaging. J Am Soc Echocardiogr. 2006;19:1494-501.

19. Qi X, Cogar B, Hsiung MC, et al. Live/real time threedimensional transthoracic echocardiographic assessment of left ventricular volumes, ejection fraction, and mass compared with magnetic resonance imaging. Echocardiography. 2007;24:166-73.

20. Jenkins $\mathrm{C}$, Leano $\mathrm{R}$, Chan J, et al. Reconstructed versus real-time 3-dimensional echocardiography: comparison with magnetic resonance imaging. J Am Soc Echocardiogr. 2007;20:862-8.

21. Soliman OI, Krenning BJ, Geleijnse ML, et al. Quantification of left ventricular volumes and function in patients with cardiomyopathies by real-time three-dimensional echocardiography: a head-to-head comparison between two different semiautomated endocardial border detection algorithms. J Am Soc Echocardiogr. 2007;20:1042-9.

22. Soliman OI, Krenning BJ, Geleijnse ML, et al. A comparison between QLAB and TomTec full volume reconstruction for real time three-dimensional echocardiographic quantification of left ventricular volumes. Echocardiography. 2007;24:967-74.

23. Krenning BJ, Kirschbaum SW, Soliman OI, et al. Comparison of contrast agent-enhanced versus non-contrast agent-enhanced realtime three-dimensional echocardiography for analysis of left ventricular systolic function. Am J Cardiol. 2007;100:1485-9.

24. Marsan NA, Bleeker GB, Ypenburg C, et al. Real-time threedimensional echocardiography permits quantification of left ventricular mechanical dyssynchrony and predicts acute response to cardiac resynchronization therapy. J Cardiovasc Electrophysiol. 2008;19:3929.

25. Mor-Avi V, Jenkins C, Kuhl HP, et al. Real-time 3-dimensional echocardiographic quantification of left ventricular volumes: multicenter study for validation with magnetic resonance imaging and investigation of sources of error. JACC Cardiovasc Imaging. 2008; 1:413-23.

26. Pouleur AC, le Polain de Waroux JB, Pasquet A, et al. Assessment of left ventricular mass and volumes by three-dimensional echocardiography in patients with or without wall motion abnormalities: comparison against cine magnetic resonance imaging. Heart. 2008;94:1050-7.

27. Bicudo LS, Tsutsui JM, Shiozaki A, et al. Value of real time threedimensional echocardiography in patients with hypertrophic cardiomyopathy: comparison with two-dimensional echocardiography and magnetic resonance imaging. Echocardiography. 2008;25:717-26.

28. Soliman OI, Kirschbaum SW, van Dalen BM, et al. Accuracy and reproducibility of quantitation of left ventricular function by realtime three-dimensional echocardiography versus cardiac magnetic resonance. Am J Cardiol. 2008;102:778-83.

29. Chukwu EO, Barasch E, Mihalatos DG, et al. Relative importance of errors in left ventricular quantitation by two-dimensional echocardiography: insights from three-dimensional echocardiography and cardiac magnetic resonance imaging. J Am Soc Echocardiogr. 2008;21:990-7. 
30. Hansegard J, Urheim S, Lunde K, et al. Semi-automated quantification of left ventricular volumes and ejection fraction by real-time three-dimensional echocardiography. Cardiovasc Ultrasound. 2009;7:18.

31. Tighe DA, Rosetti M, Vinch CS, et al. Influence of image quality on the accuracy of real time three-dimensional echocardiography to measure left ventricular volumes in unselected patients: a comparison with gated-SPECT imaging. Echocardiography. 2007;24:1073-80.

32. Corsi C, Coon P, Goonewardena S, et al. Quantification of regional left ventricular wall motion from real-time 3-dimensional echocardiography in patients with poor acoustic windows: effects of contrast enhancement tested against cardiac magnetic resonance. J Am Soc Echocardiogr. 2006;19:886-93.

33. Hare JL, Jenkins C, Nakatani S, et al. Feasibility and clinical decision-making with $3 \mathrm{D}$ echocardiography in routine practice. Heart. 2008;94(4):440-5.

34. Cleland JG, Daubert JC, Erdmann E, et al. The effect of cardiac resynchronization on morbidity and mortality in heart failure. $\mathrm{N}$ Engl J Med. 2005;352:1539-49.

35. Yu CM, Fung JW, Zhang Q, et al. Tissue Doppler imaging is superior to strain rate imaging and postsystolic shortening on the prediction of reverse remodeling in both ischemic and nonischemic heart failure after cardiac resynchronization therapy. Circulation. 2004;110:66-73.

36. Yu CM, Zhang Q, Fung JW, et al. A novel tool to assess systolic asynchrony and identify responders of cardiac resynchronization therapy by tissue synchronization imaging. J Am Coll Cardiol. 2005;45:677-84.

37. Chung ES, Leon AR, Tavazzi L, et al. Results of the Predictors of Response to CRT (PROSPECT) trial. Circulation. 2008;117:260816.

38. Kapetanakis S, Kearney MT, Siva A, et al. Real-time threedimensional echocardiography: a novel technique to quantify global left ventricular mechanical dyssynchrony. Circulation. 2005;112:9921000.

39. Burgess MI, Jenkins C, Chan J, et al. Measurement of left ventricular dyssynchrony in patients with ischaemic cardiomyopathy: a comparison of real-time three-dimensional and tissue Doppler echocardiography. Heart. 2007;93:1191-6.

40. Takeuchi M, Jacobs A, Sugeng L, et al. Assessment of left ventricular dyssynchrony with real-time 3-dimensional echocardiography: comparison with Doppler tissue imaging. J Am Soc Echocardiogr. 2007;20:1321-9.

41. Marsan NA, Bleeker GB, Ypenburg C, et al. Real-time threedimensional echocardiography as a novel approach to assess left ventricular and left atrium reverse remodeling and to predict response to cardiac resynchronization therapy. Heart Rhythm. 2008;5:1257-64.

42. Soliman OI, Van Dalen B, Nemes A, et al. Quantification of left ventricular systolic dyssynchrony by real-time three-dimensional echocardiography. J Am Soc Echocardiogr. 2009;22:232-9.

43. Kleijn SA, Van Dijk J, de Cock CC, et al. Assessment of intraventricular mechanical dyssynchrony and prediction of response to cardiac resynchronization therapy: comparison between tissue
Doppler imaging and real-time three-dimensional echocardiography. J Am Soc Echocardiogr. 2009;22:1047-54.

44. Van Dijk J, Knaapen P, Russel IK, et al. Mechanical dyssynchrony by 3D echo correlates with acute haemodynamic response to biventricular pacing in heart failure patients. Europace. 2008;10:63-8.

45. Van Dijk J, Dijkmans PA, Gotte MJ, et al. Evaluation of global left ventricular function and mechanical dyssynchrony in patients with an asymptomatic left bundle branch block: a real-time 3D echocardiography study. Eur J Echocardiogr. 2008;9:40-6.

46. Marsan NA, Henneman MM, Chen J, et al. Real-time threedimensional echocardiography as a novel approach to quantify left ventricular dyssynchrony: a comparison study with phase analysis of gated myocardial perfusion single photon emission computed tomography. J Am Soc Echocardiogr. 2008;21:801-7.

47. Delgado V, Sitges M, Vidal B, et al. Assessment of left ventricular dyssynchrony by real-time three-dimensional echocardiography. Rev Esp Cardiol. 2008;61:825-34.

48. Gimenes VM, Vieira ML, Andrade MM, et al. Standard values for real-time transthoracic three-dimensional echocardiographic dyssynchrony indexes in a normal population. J Am Soc Echocardiogr. 2008;21:1229-35.

49. Liodakis E, Sharef OA, Dawson D, et al. The use of real-time three-dimensional echocardiography for assessing mechanical synchronicity. Heart. 2009;95:1865-71.

50. Soliman OI, Geleijnse ML, Theuns DA, et al. Usefulness of left ventricular systolic dyssynchrony by real-time three-dimensional echocardiography to predict long-term response to cardiac resynchronization therapy. Am J Cardiol. 2009;103:1586-91.

51. Sonne C, Sugeng L, Takeuchi M, et al. Real-time 3-dimensional echocardiographic assessment of left ventricular dyssynchrony: pitfalls in patients with dilated cardiomyopathy. JACC Cardiovasc Imaging. 2009;2:802-12.

52. Becker M, Hoffmann R, Schmitz F, et al. Relation of optimal lead positioning as defined by three-dimensional echocardiography to long-term benefit of cardiac resynchronization. Am J Cardiol. 2007;100:1671-6.

53. Deplagne A, Lafitte S, Reuter S, et al. Absence of additional improvement in outcome of patients receiving cardiac resynchronization therapy paced at the most delayed left ventricular region. Arch Cardiovasc Dis. 2009;102:641-9.

54. Nesser HJ, Mor-Avi V, Gorissen W, et al. Quantification of left ventricular volumes using three-dimensional echocardiographic speckle tracking: comparison with MRI. Eur Heart J. 2009;30:1565-73.

55. Andrade J, Cortez LD, Campos O, et al. Left ventricular twist: comparison between two- and three-dimensional speckle-tracking echocardiography in healthy volunteers. Eur J Echocardiogr. 2011;12(1):76-9.

56. Thebault C, Donal E, Bernard A, et al. Real-time three-dimensional speckle tracking echocardiography: a novel technique to quantify global left ventricular mechanical dyssynchrony. Eur J Echocardiogr. 2011;12(1):26-32.

57. Tanaka H, Hara H, Saba S, et al. Usefulness of three-dimensional speckle tracking strain to quantify dyssynchrony and the site of latest mechanical activation. Am J Cardiol. 2010;105(2):235-42. 